\section{Intestinal permeability: the cellobiose/mannitol test}

EDIToR,-I should like to bring to your attention a conceptual error in the paper by Daniele et al (Gut 2001;48:28-33) regarding the cellobiose/mannitol test. The authors suggest that improvement in the cellobiose/ mannitol ratio reflects improvement in permeability from the use of oral glutamine. However, only mannitol excretion improved significantly with glutamine; cellobiose excretion remained unchanged. As the authors explain in their methods section, it is the increased cellobiose excretion that reflects increased permeability, not the decrement in mannitol excretion. Therefore, modifications in sugar transport induced by 5 -fluorouraci (5-FU) reflected only an absorptive, not a permeability, defect. The decrement in mannito excretion parallels the decrement in D-xylose excretion, probably reflecting decreased transcellular passage of the test sugars induced by 5-FU and improved with glutamine.

R M CRAIG

Department of Gastroenterology and Hepatology, Northwestern University, Chicago, Illinois 60611, USA r-craig@northwestern.edu

\section{Reply}

EDIToR,-I thank Dr Craig for raising this issue but I do not see any conceptual error. The apparent inconsistency that he points out in our paper (Gut 2001;48:28-33) is due to the controversy surrounding transcellular permeation of mannitol, as well as of other monosaccharides. ${ }^{1}$ While transcellular permeation of mannitol is well known, its use for osmotic shrinkage of membrane vescicles and as an extracellular fluid volume marker suggests that, at least in part, mannito diffuses through the intercellular tight junctions. Thus it seems justified talking of permeability for mannitol. One of the reasons for its use in combination with cellobiose is the different molecular sizes of the two probes: the smaller size of mannitol allows its passage through the small tight junctions of the villi while the larger cellobiose passes through the larger tight junctions of the crypts.

Finally, we did find an increase in cellobiose excretion after fluorouracil ( $5-\mathrm{FU})$ that was in part prevented by oral glutamine. Although this difference did not reach statistical significance, overall the data indicate increased intestinal permeability after $5-\mathrm{FU}$, partially prevented by oral glutamine.

B DANIELE Divisione Di Oncologia Medica B, Istituto Nazionale Tumori, via M Semmola, 80131 Napoli, Italy bdaniele@sirio-oncology.it

1 Bjarnason I, Macpherson AJ, Hollander D. Intestinal permeability: an overview. Gastroenterology 1995;108:1566-81.

2 Kessler M, Acuto O, Storelli C, et al. A modified procedure for the rapid preparation of efficiently transporting vescicles from small intestinal brush border membranes. Biochim Biophys Acta 1978;506:136-54.

\section{Evaluation of the role of CFTR in alcohol related pancreatic disease}

EDIToR,-In up to $30 \%$ of patients with idiopathic pancreatitis (IP) a mutation of at least one or both alleles of the cystic fibrosis transmembrane conductance regulator (CFTR) gene can be identified. ${ }^{1-3}$ The study by Malats

et al (Gut 2001;48:70-4) addressed the question of whether CFTR mutations, possibly together with environmental factors such as alcohol, may be associated with chronic pancreatitis or pancreatic cancer. The vast majority of the pancreatic patients $(86.4 \%)$ investigated by Malats et al were diagnosed as having alcoholic pancreatitis (AP), and $75.4 \%$ of the cancer patients were daily drinkers. The authors found no statistically significant difference in the prevalence of delta-F508 $(0 \% ; 2.4 \%)$ and the $5 \mathrm{~T}$ allele $(10.5 \% ; 5.5 \%)$ in the AP or cancer groups compared with the expected prevalence in the general population. The lack of a positive association of both delta-F508 and the 5T allele with $\mathrm{AP}$ is neither surprising nor argues against involvement of CFTR variations in the development of AP, considering the following.

In cystic fibrosis $(\mathrm{CF})$, the degree of correlation between CFTR genotype and CF phenotype varies between clinical components but is highest for pancreatic involvement. ${ }^{4}$ CFTR mutations can simplify be divided into "severe" and "mild" with respect to the degree to which mutations impair CFTR function. ${ }^{5}$ Approximately $85 \%$ of CF patients suffer from pancreatic insufficiency (PI) while $\sim 15 \%$ are pancreatic sufficient (PS). Generally patients with PI carry two "severe" mutations whereas PS is associated with at least one "mild" mutation (fig 1). In CF, pancreatitis is seen rather frequently in PS patients but not in PI patients. Today, more than $850 \mathrm{CF}$ mutations have been reported to the CF Consortium (httplwww.genet. sickkids.on.ca/cftr). The deletion delta-F508, accounting for about $70 \%$ of mutant $\mathrm{CF}$ alleles worldwide and approximately $53 \%$ in Spain, studied by Malats $e t a l$, is responsible for severe functional loss of CFTR function. Three additional studies on the prevalence of an abnormal CFTR allele in AP have been published as full papers. ${ }^{367}$ Pooling these four studies, one or two mutant CFTR alleles were detected in $9 / 217(4.1 \%)$ patients with AP. But the detection rate varies between $0 \%$ and $8.5 \%$ depending on the sensitivity of the screening method to detect an abnormal CF allele in the corresponding population (53$94 \%)$. None of the studies revealed a positive association of the $5 \mathrm{~T}$ allele with IP or AP. Compared with the general population, delta-F508 was significantly more frequent in

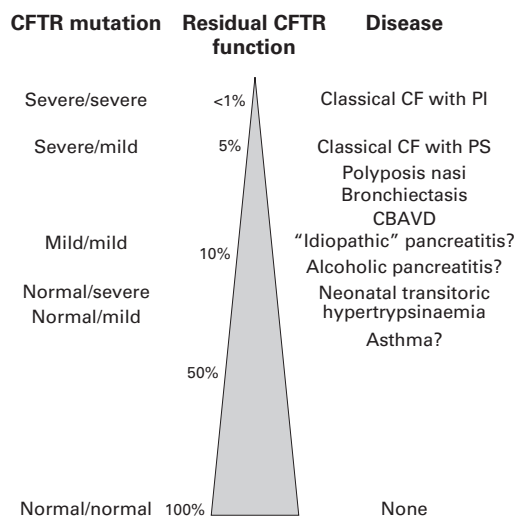

Figure 1 Disease manifestation according to residual cystic fibrosis transmembrane conductance regulator (CFTR) function as a result of the combination of severe or mild CFTR genotype. CF, cystic fibrosis; PS, pancreatic sufficiency; PI, pancreatic insufficiency; $C B A V D$, congenital absence of the vas deferens.
British and US Caucasian, but not in Australian or Spanish AP patients.

Up to now no environmental or genetic cofactor was identified in patients with mutant CFTR alleles associated IP, suggesting that impairment of CFTR function alone may be enough to induce pancreatitis. ${ }^{12389}$ On the other hand it may be speculated that patients with an abnormal CFTR allele, who develop pancreatitis in conjunction with alcohol abuse, may be characterised by a higher residual CFTR function, which by itself is not capable of inducing pancreatitis.

Therefore, to delineate the genetic background of pancreatic disease in AP it seems to be more appropriate to investigate the prevalence of uncommon mild variants ("atypical mutations") in large cohorts of AP patients than to test for the more common ("severe, typical") mutations of the CFTR gene in small patient groups. It has to be considered that the test kits for CFTR mutations often used in routine screening are usually designed to detect the more severe CF mutations. This would result in missing a substantial number of patients with milder CFTR mutations, as suggested by preliminary data on more comprehensive genetic testing in patients with ICP.

J OCKENGA

Department of Gastroenterology, Medical School Fannover, 30625 Hannover, Germany M STUHRMANN Department of Human Genetics, Medical School Hannover, 30625 Hannover, Germany M P MANNS

Department of Gastroenterology, Medical School Hannover, 30625 Hannover, Germany

Correspondence to: Dr J Ockenga.

Ockenga.Johann@mh-hannover.de

1 Cohn JA, Friedman KJ, Noone PG, et al. Relation between mutations of the cystic fibrosis gene and idiopathic pancreatitis. $N$ Engl F Med 1998;339:653-8.

2 Ockenga J, Stuhrmann M, Ballmann M, et al. Mutations of the cystic fibrosis gene, but not cationic trypsinogen gene, are associated with recurrent or chronic idiopathic pancreatitis. Am ₹ Gastroenterol 2000;95:2061-9.

3 Sharer N, Schwarz M, Malone G, et al. Mutations of the cystic fibrosis gene in patients Mutations of the cystic fibrosis gene in patients
with chronic pancreatitis. $N$ Engl $\mathcal{F}$ Med 1998;339:645-52.

4 Stuhrmann M, Macek M Jr, Reis A, et al. Genotype analysis of cystic fibrosis patients in relation to pancreatic sufficiency. Lancet 1990; 335:738-9

5 Zielenski J. Genotypic and phenotype in cystic fibrosis. Respiration 2000;67:117-33.

6 Monaghan KG, Jackson CE, KuKuruga DL, et al. Mutation analysis of the cystic fibrosis and cationic trypsinogen genes in patients with alcohol-related pancreatitis. Am $\mathcal{F}$ Med Genet 2000;94:120-4

7 Norton ID, Apte MV, Dixson H, et al. Cystic fibrosis genotypes and alcoholic pancreatitis. $\mathcal{F}$ Gastroenterol Hepatol 1998;13:496-9.

8 Cohn JA, Bornstein JD, Jowell PS. Cystic fibrosis mutations and genetic predisposition to idiopathic chronic pancreatitis. Med Clin North Am 2000;84:621-31.

9 Ockenga J, Dork T, Stuhrmann M. Low prevalence of SPINK1 gene mutations in adult patients with chronic idiopathic pancreatitis. $\mathcal{f}$ Med Genet 2001;38:243-4.

\section{Reply}

EDITOR,-We agree with the view of Ockenga et al that from an ideal research perspective a complete analysis of the cystic fibrosis transmembrane conductance regulator (CFTR) gene should be performed for cases of pancreatitis before a definitive statement on 
the role of this gene in chronic pancreatitis can be made. However, it is well known that $18-30 \%$ of patients with CFTR related disorders (congenital bilateral absence of the vas deferens and bronchiectasis) have only one CFTR mutated allele. ${ }^{1-3}$ Thus despite our study being based on only the two most common CFTR mutations (F508del and 5T), these two alterations should suffice to rule out or confirm a potential role of CFTR in patients with chronic pancreatic diseases. Furthermore, complete analysis of CFTR in the general population has led to the identification of amino acid variants of yet unknown functional significance in about $10 \%$ of subjects. ${ }^{4}$ It is highly likely that complete analysis of CFTR would render a large number of amino acid changes of uncertain clinical and functional consequences, as it has been shown for patients with asthma. ${ }^{5}$ As we proposed in our paper (Gut 2001;48:70-4), only the design of large studies specifically addressing these issues in target and adequate control populations and a comprehensive molecular analysis of CFTR will answer the question on the role of this gene in chronic pancreatic disease.

X ESTIVILL T CASALS Centre de Genètica Mèdica i Molecular-IRO, L'Hospitalet de Llobregat, Barcelona, Spain N MALATS M PORTA Grup de Recerca d'Epidemiologia Clínica $i$ Molecular del Càncer, Institut Municipal d'Investigació Mèdica, Universitat Pompeu Fabra, Barcelona, Universitat Autònoma de Barcelona, Spain

L GUARNER Servei de Digestiu, Hospital Vall d'Hebron, Barcelona, Spain

F X REAL

Unitat de Biologia Cel.lular i Molecular, Institut Municipal d'Investigació Mèdica, Universitat Pompeu Fabra, Barcelona, Spain

Correspondence to Dr Malats.

nuria@imim.es

1 Casals T, Bassas L1, Egozcue S, et al. Heterogeneity for mutations in the CFTR gene and clinical correlations in patients with congenital absence of the vas deferens. Hum Reprod absence of the

2 Pignatti PF, Bombieri C, Benetazzo M, et al. CFTR gene variant IVS8-5T in disseminated bronchiectasis. Am f Hum Genet 1996;58:88992 .

3 Girodon E, Cazeneuve C, Lebargy F, et al. CFTR gene mutations in adults with disseminated bronchiectasis. Eur f Hum Genet 1997;5 149-55.

4 Bombieri C, Giorgi S, Carles S, et al. A new approach for identifying non-pathogenic mutations. An analysis of cystic fibrosis transmembrane regulator gene in normal individuals. Hum Genet 2000;106:172-8.

5 Lázaro C, de Cid R, Sunyer J, et al. Missense mutations in the cystic fibrosis gene in adult patients with asthma. Hum Mutat 1999;14: 510-19.

\section{Alcohol, obesity, and TNF- $\alpha$}

EDITOR,- The conclusions reached by Wigg et al (Gut 2001;48:206-11) about the origin and importance of tumour necrosis factor $\alpha$ (TNF- $\alpha$ ) in non-alcoholic steatohepatitis (NASH) patients have failed to take into account the relationship between even modest alcohol consumption and $\mathrm{TNF}-\alpha$ production and function. The authors found a lack of correlation between obesity and $\mathrm{TNF}-\alpha$ levels in NASH patients and concluded that TNF- $\alpha$, which they see as central to the pathogenesis of the condition, must have other sources.
We first described the strong correlation between obesity and serum TNF- $\alpha$ in $1998 .{ }^{1}$ Adipose tissue synthesises a number of proinflammatory cytokines. ${ }^{2}$ The negative correlation found in the Adelaide study is surprising given the findings in larger studies of non-NASH subjects and may be due to the small study numbers and not correcting for modest alcohol intake.

Alcohol consumption is considered a risk factor for the development and progression of liver disease in patients with fatty livers. We previously showed a strong negative correlation between any alcohol consumption and serum TNF- $\alpha$ levels in a general population sample. Modest alcohol consumption is known to suppress $\mathrm{TNF}-\alpha$ production by monocytes, probably by suppressing posttranscriptional TNF- $\alpha$ production. ${ }^{3}$ Furthermore, alcohol also has effects on $\mathrm{TNF}-\alpha$ function mediated via high density lipoprotein (HDL). Alcohol enhances HDL levels by stimulating lipoprotein lipase activity in adipose tissue. ${ }^{4}$ HDL not only inhibits TNF- $\alpha$ release from macrophages ${ }^{5}$ but also protects certain cells against $\mathrm{TNF}-\alpha$ induced damage. $^{6}$

If $\mathrm{TNF}-\alpha$ is important, then modest alcohol intake should be protective via suppression of TNF- $\alpha$. This raises the possibility that $\mathrm{TNF}-\alpha$ is not important in early steatohepatitis

In defining patients with $\mathrm{NASH}$, alcohol consumption must be rigorously excluded. In the Adelaide study, 10 of 22 patients drank up to $20 \mathrm{~g}$ of alcohol per day; however, even modest amounts of alcohol have effects on TNF- $\alpha$ levels and function.

The known interaction between alcohol and obesity in the pathogenesis of fatty liver and steatohepatitis suggests that investigators must look to factors other than TNF- $\alpha$ in studying the early pathogenesis of this condition. In the same way that altered cytokine homeostasis has been implicated in alcoholic liver disease, NASH is probably caused by changes to more than one proinflammatory cytokine. Interleukin 6 (IL-6) is a proinflammatory cytokine, a hepatocyte stimulating factor, and inhibitor of hepatic apoptosis. It has been suggested that hepatic steatosis is due to the rate of hepatocyte apoptosis becoming insufficient to match the rate of hepatocyte proliferation. ${ }^{7}$ IL- 6 induced liver regeneration may render the liver more susceptible to the effects of other insults. Unlike TNF- $\alpha$, serum IL- 6 exhibits a positive correlation with both obesity and alcohol intake (fig 1). ${ }^{18}$ So far IL-6 has not been studied in the aetiology of NASH.
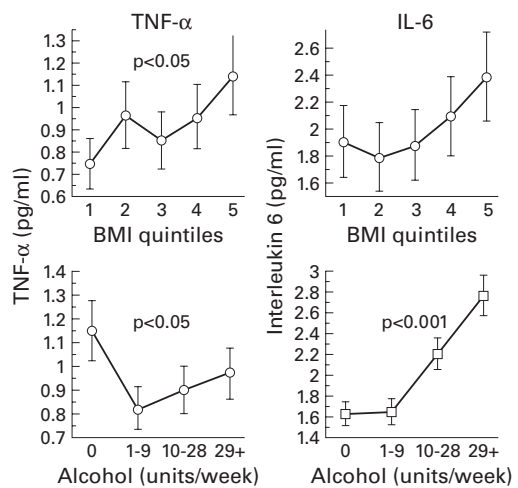

Figure 1 Relationship between the cytokines tumour necrosis factor $a(T N F-\alpha)$ and interleukin 6 (IL-6), and obesity and alcohol. $B M I$, body mass index.
Future studies examining the link between TNF- $\alpha$ and NASH will need to rigorously control for alcohol consumption and assess many other aspects of the inflammatory cytokine network.

A POULLIS

M A MENDALL

Mayday University Hospital,

Thornton Heath, Surrey, UK

Correspondence to: M A Mendall.

mike.mendall@mhc-tr.sthames.nhs.uk

1 Mendall MA, Patel P, Asante M, et al. Relation of serum cytokine concentrations to cardiovascular risk factors and coronary heart disease. Heart 1997;78:273-7.

2 Mendall MA, Patel P, Ballam L, et al. C Reactive protein and its relation to cardiovascular risk factors: a population based cross sectional study. BMF 1996;312:1061-5.

3 Zhang Z, Cork J, Ye P, et al. Inhibition of TNFalpha processing and TACE-mediated ectodomain shedding by ethanol. F Leukoc Biol 2000; 67:856-62.

4 Taskinen MR, Nikkila EA, Valimaki M, et al. Alcohol induced changes in serum lipoproteins and in their metabolism. Am Heart f 1987;113: 458-64.

5 Giorna J, La Ville AE, Hermas M, et al. Oxidized lipoproteins including HDL and their lipid peroxidation products inhibit TNF-alpha secretion by THP-1 human macrophages. Free Radic Biol Med 1997;23:658-67.

6 Sugano M, Tsuchida K, Makino N. Highdensity lipoproteins protect endothelial cells density lipoproteins protect endothelial cells
from TNF-alpha-induced apoptosis. Biochem from TNF-alpha-induced apoptosis.
Biophys Res Commun 2000;272:872-6.

7 Tilg H, Diehl AM. Mechanisms of disease: Cytokines in alcoholic and non-alcoholic steatohepatitis. N Engl f Med 2000;343:1467-76.

8 Yudkin JS, Kumari M, Humphries SE, et al. Inflammation, obesity, stress and coronary sclerosis 2000;148:209-14.

\section{Reply}

EDITOR,-Our recent paper found increased small bowel bacterial overgrowth (50\% versus $22 \%$ ) and twofold increased systemic levels of tumour necrosis factor $\alpha$ (TNF- $\alpha)$ in patients with non-alcoholic steatohepatitis (NASH) compared with control age and sex matched subjects (Gut 2001;48:206-11). Poullis and Mendall question the finding of elevated TNF- $\alpha$ levels in blood in NASH subjects and quote their own work of elevated TNF- $\alpha$ levels in obese, male, middle aged subjects. ${ }^{1}$ There was no correlation between TNF- $\alpha$ levels and obesity in our study whereas their study showed a correlation with obesity. How can this be explained? The question comes down to whether TNF- $\alpha$ is being produced predominantly in adipose tissue or in the liver, and which of these contributes to elevated systemic levels. At the moment this cannot be resolved. TNF- $\alpha$ will need to be investigated in liver biopsies and TNF- $\alpha$ levels sampled from the hepatic vein (not entirely impossible). The same should be done in animal models of obesity. In the meantime, it would be important to ascertain what proportion of obese patients have unrecognised NASH and whether this could explain the elevated TNF- $\alpha$ levels in obesity. Several lines of evidence suggest $\mathrm{TNF}^{-} \alpha$ is upregulated in the liver in alcoholic liver disease and presumably this is reflected by raised serum levels. We doubt therefore whether a low $(<20 \mathrm{~g} /$ day) consumption of alcohol reduces systemic TNF- $\alpha$ levels but this could be formally studied. We have re-examined our data and found that there is no difference in mean TNF- $\alpha$ levels between those who 